\title{
Prediction of Static Aerodynamic Hysteresis on a Thin Airfoil Using OpenFOAM
}

\author{
Mohamed Sereez, ${ }^{*}$ Nikolay B. Abramov ${ }^{\dagger}$ and Mikhail G. Goman ${ }^{\ddagger}$ \\ De Montfort University, Leicester, LE1 9BH, United Kingdom
}

\begin{abstract}
The paper presents computational prediction of aerodynamic hysteresis loops in static conditions for a two-dimensional aerofoil which was used as a cross-section profile for a rectangular wing with an aspect ratio of five, tested in the TsAGI T-106 wind tunnel at Reynolds number, $R e=6 \times 10^{6}$ and Mach number, $M=0.15$. Tests in the wind tunnel showed that minor changes in the curvature of the leading edge of the thin aerodynamic profile lead to a significant increase in the maximum lift coefficient when significant hysteresis loops appear in the aerodynamic characteristics of the wing. The computational predictions of stall aerodynamics presented in this paper are made for a two-dimensional profile using the OpenFOAM open-source code to simulate a flow based on the unsteady Reynolds averaged Navier-Stokes equations using the Spalart-Allmaras turbulence model. The calculation results confirm the existence of loops of static aerodynamic hysteresis and bistable structures of the separated flow, and the results are qualitatively similar to the results observed experimentally on the wing with a finite aspect ratio.
\end{abstract}

\section{Nomenclature}

$$
\begin{array}{ll}
C_{L}, C_{D}, C_{M} & =\text { lift, drag, and pitching moment coefficients } \\
\operatorname{CoM}, \operatorname{CoG}, \operatorname{CoP} & =\text { centers of moment, gravity, and pressure } \\
C_{\text {ref }}, S_{\text {ref }} & =\text { reference chord length and reference area } \\
k & =\text { reduced frequency, } \omega c /\left(2 U_{\text {ref }}\right) \\
p & =\text { static pressure } \\
R e & =\text { Reynolds number } \\
|S| & =\text { strain rate magnitude } \\
S c_{t} & =\text { turbulent Schmidt number } \\
S_{r} & =\text { Strouhal number } \\
t & =\text { physical time }
\end{array}
$$

\footnotetext{
* Research Fellow, School of Engineering and Sustainable Development.

†Senior Research Fellow, School of Engineering and Sustainable Development.

†Professor of Dynamics, School of Engineering and Sustainable Development, FRAeS and Senior Member AIAA.
} 


$\begin{array}{ll}U & =\text { flow velocity } \\ U_{\text {ref }} & =\text { reference flow velocity } \\ \alpha & =\text { angle of attack } \\ \alpha_{s} & =\text { stall angle of attack } \\ \mu & =\text { dynamic viscosity } \\ \mu_{t} & =\text { turbulent viscosity } \\ v & =\text { kinematic viscosity } \\ \hat{v}_{t} & =\text { modified turbulent viscosity } \\ \tau_{i j} & =\text { Reynolds stress tensor }\end{array}$

\section{Introduction}

ERodynamic hysteresis in the stall region can be observed under both static and dynamic conditions [1, 2]. $k>0.05$ were widely studied and discussed in the literature [2, 3]. The static aerodynamic hysteresis observed in slow-motion typical for low frequencies $k<0.05$ is a more complex phenomenon, and it is still not fully understood. For example, at low Reynolds numbers, it can be affected by both a laminar separation bubble near the leading edge and a flow separation extending forward from the trailing edge; for large Reynolds numbers, lower importance of the separation bubble can be expected. Under static conditions, this phenomenon exhibits a very high sensitivity to the reduced frequency, the Reynolds number, and the leading edge curvature [1]. Two or more separated flow structures with different topologies generate substantially different aerodynamic forces and moments at the same angle of attack. In the presence of static hysteresis, the aerodynamic characteristics depend on the direction of change of the angle of attack, one of the manifestations of which is the presence of abrupt jumps at critical points. Under dynamic conditions, the hysteresis loops become wider due to delayed transitions between different branches.

The prediction of aerodynamic hysteresis in the stall region, either in wind tunnel tests or via computational simulations, is a difficult task owing to the strong sensitivity of stall aerodynamics to various physical and computational factors. The intensive flow turbulence in the wind tunnel and the vibrations of the sting supporting the test model may induce premature transitions between static hysteresis branches. In computational simulations, there is a strong sensitivity to the numerical solver, grid resolution, turbulence model, etc. [3]. Thus, it is important to improve understanding of the physical mechanisms of this phenomenon in support of more reliable modeling of stall aerodynamics. Examples range from wind turbines and micro air vehicles operating at low Reynolds numbers to the problem of loss of control in-flight (LOC-I) of transport aircraft, for which modeling of stall/post-stall aerodynamics at high Reynolds numbers is required [4]. 
There have been a number of discussions of the static hysteresis phenomenon in the literature for relatively low Reynolds numbers $R e<10^{6}[5]-8$, but limited information is available about the phenomenon at higher Reynolds numbers $R e \geq 10^{6}$ (see, e.g., [9]). Published results show that the widths of static hysteresis loops depend on, among other things, the aspect ratio of the wing, the leading edge shape, and the Reynolds number [7, 9]. Static hysteresis may disappear under the action of external disturbances, for example, when turbulence in a wind tunnel is high [8]. Often, experimentally determined dependences of aerodynamic loads in the stall region show abrupt jumps, indicating the possible existence of hysteresis loops, which could be identified if the tests were run with the reverse variation of the angle of attack. Therefore, the identification of static hysteresis dependences needs particular attention and the application of special methods in both experimental and computational approaches. It is very important to develop such methods because not only different wing planforms but also full aircraft configurations are prone to this phenomenon in the stall region.

The work by Mittal and Saxena [10, 11] on flow past the NACA 0012 airfoil at $R e=1 \times 10^{6}$ probably was the first successful attempt to show the existence of static aerodynamic hysteresis by computational simulation. In their computational framework, the unsteady Reynolds-Averaged Navier-Stokes (URANS) equations were employed using a stabilized finite element method (FEM) along with the algebraic Baldwin-Lomax (BL) model for turbulence closure. Bi-stable flow structures were identified for two angles of attack: $\alpha=17^{\circ}$ and $\alpha=18.5^{\circ}$ so that the flow structure on the top branch of the static hysteresis was characterized by shedding of small-scale vortices with high frequency, and the bottom branch was characterized by shedding of large-scale vortices with relatively lower frequency. The averaged value of the lift coefficient, $\bar{C}_{L}$, in the top branch was much higher than that in the bottom branch. In [12], a computational prediction of the static hysteresis phenomenon was made for the NACA 0018 airfoil using the $k-\omega$ SST turbulence model. The prediction was close to the experimental results for $R e=0.3 \times 10^{6}$ presented in [5, 6, 12], but was not satisfactory for $R e=0.7 \times 10^{6}$. In a recent study [13], a comparative analysis of transition turbulence models was carried out with the objective of capturing aerodynamic static hysteresis. It was shown that the $\gamma$ and $\gamma-R e_{\theta}$ turbulence models predict the laminar bubble on the top branch rather well at $R e=0.3 \times 10^{6}$, giving a small overshoot in $C_{L \max }$, but they are completely unable to predict the lower branch of the static aerodynamic hysteresis with fully developed flow separation.

This paper presents a computational prediction of the static aerodynamic hysteresis phenomenon at relatively high Reynolds numbers $R e \approx 6.0 \times 10^{6}$ using the open-source code OpenFOAM for computational fluid dynamics (CFD) simulations, which is now widely used in both industry and academia and has recently been thoroughly validated for two- and three-dimensional flows with the Spalart-Allamaras (SA) turbulence model [14]. The work is inspired by wind tunnel test data from the TsAGI report [9], published in 1946, which include very pronounced static aerodynamic hysteresis-type dependences. In that study, a finite-aspect-ratio rectangular wing with $A R=5$ was tested in the T-106 pressurized transonic wind tunnel at TsAGI over a broad range of Reynolds numbers $R e \approx(1-6) \times 10^{6}$ and Mach numbers $M=0.15-0.45$. The wing cross-sections were formed by the TsAGI-9140 thin asymmetric profile together 
with modifications to the curvature of the leading edge by including a circular protrusion in order to increase the maximum lift coefficient $C_{L \max }$. An important feature of the aerodynamic data thereby obtained is that an increase in the Reynolds number leading to an increase in the maximum lift coefficient is accompanied by appearance and widening static aerodynamic hysteresis loops.

The remainder of this paper is structured as follows. The wind tunnel data obtained in the TsAGI variable-pressure transonic wind tunnel are presented and discussed in Sec. II The computational framework, including the governing equations, the SA turbulence model [15], mesh convergence, and numerical setup, is presented in Sec. III] Validation of the OpenFOAM for NACA 0012 airfoil at $R e=6 \times 10^{6}$ and qualitative comparison of the obtained two-dimensional computational results with the TsAGI wind tunnel data is discussed in Sec.IV] The concluding remarks are presented in Sec. V.

\section{Experimental Results}

In the mid 1940s, in the period preceding the era of supersonic flight, there was much interest in modeling the aerodynamics of thin wings, which are more suitable for supersonic flight. However, thin wings cause stall at low angles of attack, thereby posing a problem for take-off and landing. To address this, a rectangular wing with an aspect ratio $A R=5$ and thickness 9\% was tested in the T-106 variable-pressure transonic wind tunnel at TsAGI (www.tsagi.com), Russia [9]. The test conditions were in the range $R e \approx(1-6) \times 10^{6}$ and $M=0.15-0.45$, which was made possible by variation of the pressure $p=1-6 \mathrm{~atm}$. The nonsymmetric TsAGI-9140 airfoil that was used is shown in Fig. 1 1 along with a table of the airfoil coordinates. The modifications of the leading edge curvature in the form of small circular bumps tangential to the leading edge $(1 \%, 1.5 \%$, and $2 \%)$ are shown in Fig. 2 .

Figure 3 shows the wind tunnel test results for the clean and the 1.5\% modified TsAGI-9140 airfoil at two different Reynolds numbers $R e=1.0 \times 10^{6}$ and $R e \approx 5 \times 10^{6}$. The effect of the $1.5 \%$ circular protrusion at the leading edge of the airfoil is much more pronounced at the Reynolds number of $4.82 \times 10^{6}$ than at $R e=1.0 \times 10^{6}$ (see $\Delta C_{L \max }$ in Fig. 3). At $R e=4.82 \times 10^{6}$ there is a significant increase in the maximum lift coefficient $C_{L \max }$ with the delay to a higher angle of attack $\alpha_{C_{L \max }}$. At the same time the increase in $C_{L \max }$ is accompanied by the onset of a 7-degree-wide hysteresis loop.

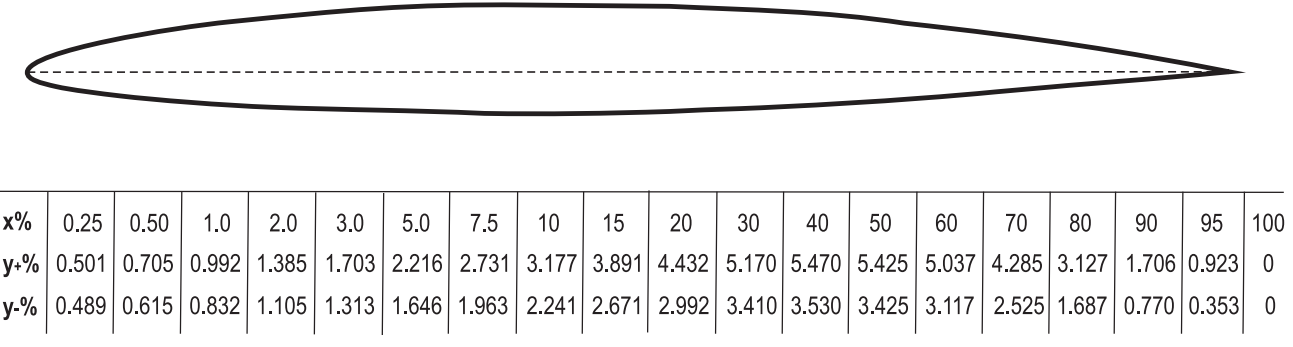

Fig. 1 TsAGI-9140 airfoil geometry.

The leading edge modifications (1.5\% and $2 \%$ ) generate significant increases in $C_{L \max }$ with the appearance of static 
hysteresis loops. For $2 \%$ modification, the static hysteresis loop is slightly shifted up and it includes an additional intermediate branch in the range $\alpha=23^{\circ}-25^{\circ}$, which presumably can be connected with the onset of an asymmetric stall on the wing (see Figure 4). When tested at higher Mach numbers $M=0.3-0.45$ with the same Reynolds number $R e=5 \times 10^{6}$, the static hysteresis loops and the increase in maximum lift practically disappear. The latter effect is not considered in this paper and needs a special investigation.

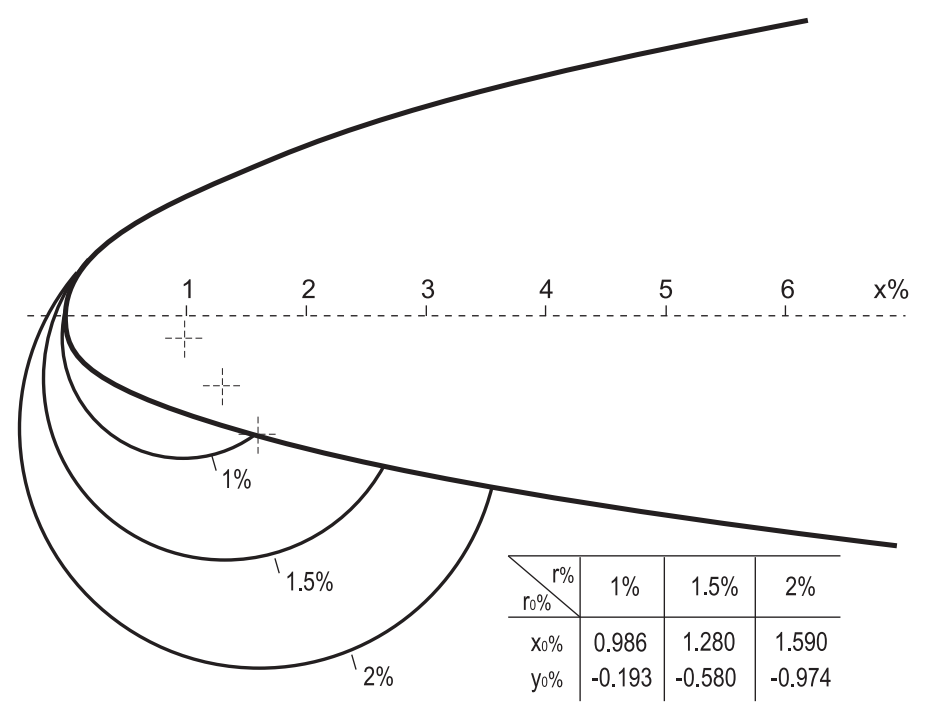

Fig. 2 Leading edge modifications of the TsAGI-9140 airfoil.

The computational predictions of static aerodynamic hysteresis in the following sections will focus only on the two-dimensional flow around the TsAGI-9140 aerodynamic profile. This was motivated by the fact that the test data of a three-dimensional wing with a separated flow can be affected by the effect of interference with the perforated cylindrical wall in the working section of the wind tunnel $(D=2.48 \mathrm{~m}, b=1.5 \mathrm{~m})$, which requires further research.

\section{Computational Framework}

Computational simulations of the two-dimensional flow around the TsAGI-9140 airfoil with its $1.5 \%$ and $2.0 \%$ leading-edge modifications under stall conditions are carried out at Reynolds numbers $R e \approx 6 \times 10^{6}$ using the open-source CFD code OpenFOAM, which is based on the finite volume method (FVM) [16, 17]. Prediction of the static aerodynamic hysteresis is conducted using the Spalart-Allamaras (SA) turbulence model [15], which is one of the turbulence models that are widely used in the aerospace industry [14].

To capture static aerodynamic hysteresis, the angle of attack is increased in small steps until the development of fully separated flow conditions and transition to the lower branch of the aerodynamic dependence. Following this transition, the angle of attack is decreased in small steps until the reattachment of the flow to the airfoil. The governing equations, mesh resolution, and turbulence model employed in the prediction of static hysteresis loops are discussed in Secs. III.A III.D The flow parameters are summarized in Table 1 


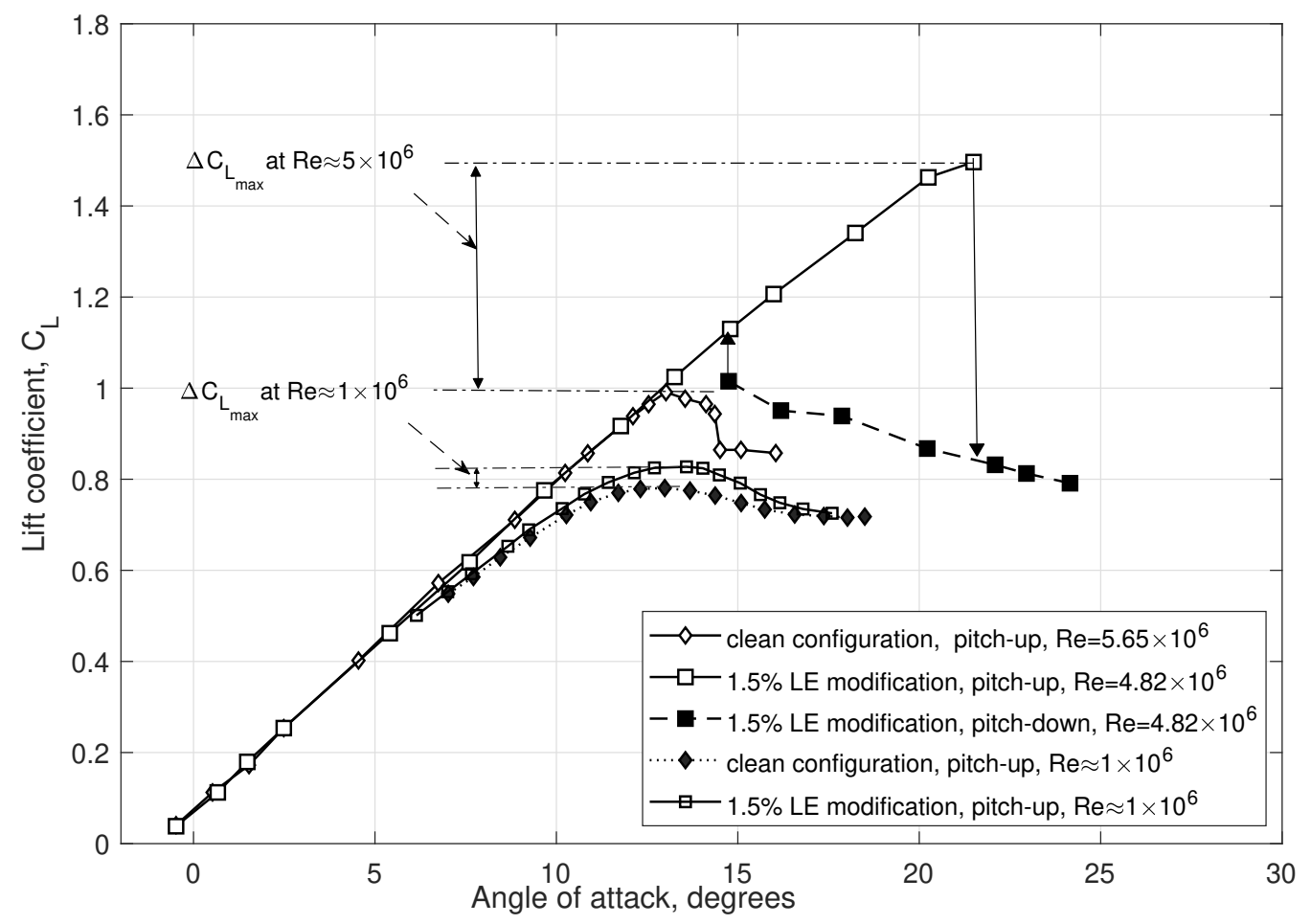

Fig. 3 The Reynolds number effect on the lift coefficient for the TsAGI-9140 wing $(A R=5)$ in clean and $1.5 \%$ leading edge modifications at $M=0.15[\overline{9}]$.

Table 1 Flow parameters

\begin{tabular}{cccc}
\hline \hline$M$ & $R e$ & $U_{\text {ref }}, \mathrm{m} / \mathrm{s}$ & $L_{\text {ref }}, \mathrm{m}$ \\
\hline 0.15 & $(4.82-5.65) \times 10^{6}$ & 51.45 & 1.45 \\
\hline \hline
\end{tabular}

\section{A. Grid and Boundary Conditions}

The airfoil is placed 30 chord lengths away from the far-field in the streamwise and orthogonal directions. The grids are produced in ANSYS ICEM CFD software using a structured meshing approach, which is suitable for grid generation around the airfoil leading edge modifications (Fig. 2). The generated grids have $Y+\leq 1$ and about 100 cells along the airfoil chord. This helps to maintain a low aspect ratio and high-quality quadrilaterals. The grid includes 45, 000 cells, and this allows a reasonably accurate simulation of the flow field. The generated grid for the $1.5 \%$ leading edge modification is shown in Fig. 5 The boundary conditions are defined as $U_{\text {wall }}=0, \hat{v}_{\text {wall }}=0, \hat{v}_{\text {farfield }}=3 v_{\text {to }}$ to $5 v_{t \infty}$, and $v_{t \text { wall }} \approx 0$ and $\partial p /\left.\partial x\right|_{\text {wall,farfield }}=0$, where $U$ is the velocity, $v_{t}$ is the turbulent viscosity, $\hat{v}$ is the modified turbulent viscosity, and $\partial p / \partial x$ is the pressure gradient. 


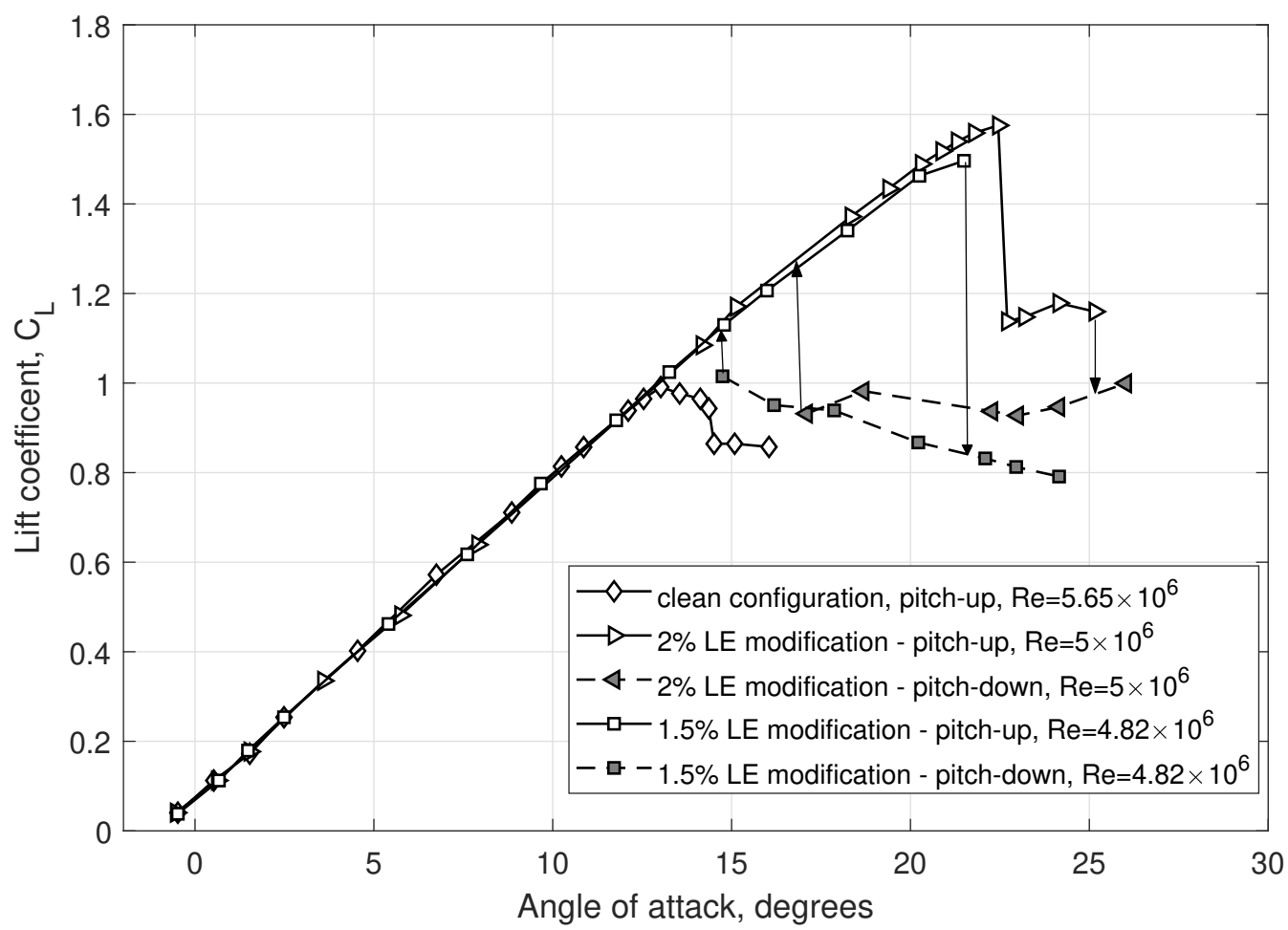

Fig. 4 Static hysteresis loops in the lift coefficient of the TsAGI-9140 wing $(A R=5)$ for $1.5 \%$ and $2 \%$ leading edge modifications at $M=0.15[9]$.

\section{B. Governing Equations}

The Navier-Stokes (NS) equations governing incompressible fluid flow are the continuity equation

$$
\nabla \cdot \mathbf{u}=0
$$

and the momentum equation

$$
\frac{\partial \mathbf{u}}{\partial t}+(\mathbf{u} \cdot \nabla) \mathbf{u}-v \nabla^{2} \mathbf{u}=-\frac{\nabla \mathbf{p}}{\rho}
$$

For flow conditions with high Reynolds numbers, the computational resources required for direct numerical simulation (DNS) of Eqs. (1) and (2) usually exceed currently available capabilities. The effect of turbulence is normally simplified by solving the unsteady Reynolds-averaged Navier-Stokes (URANS) equations, which are a time-averaged approximation of the NS equations. The averaging of the fluctuating velocities generates additional terms, known as Reynolds stresses. To describe these stresses, additional empirical equations, either algebraic or differential, are required to close the computational model. The majority of URANS turbulence models are based on the concept of eddy viscosity, equivalent to the kinematic viscosity of the fluid and describing the turbulent mixing or diffusion of the flow momentum [17]. The Reynolds stresses appearing in the URANS equations due to averaging are modeled in linear turbulence models with the 


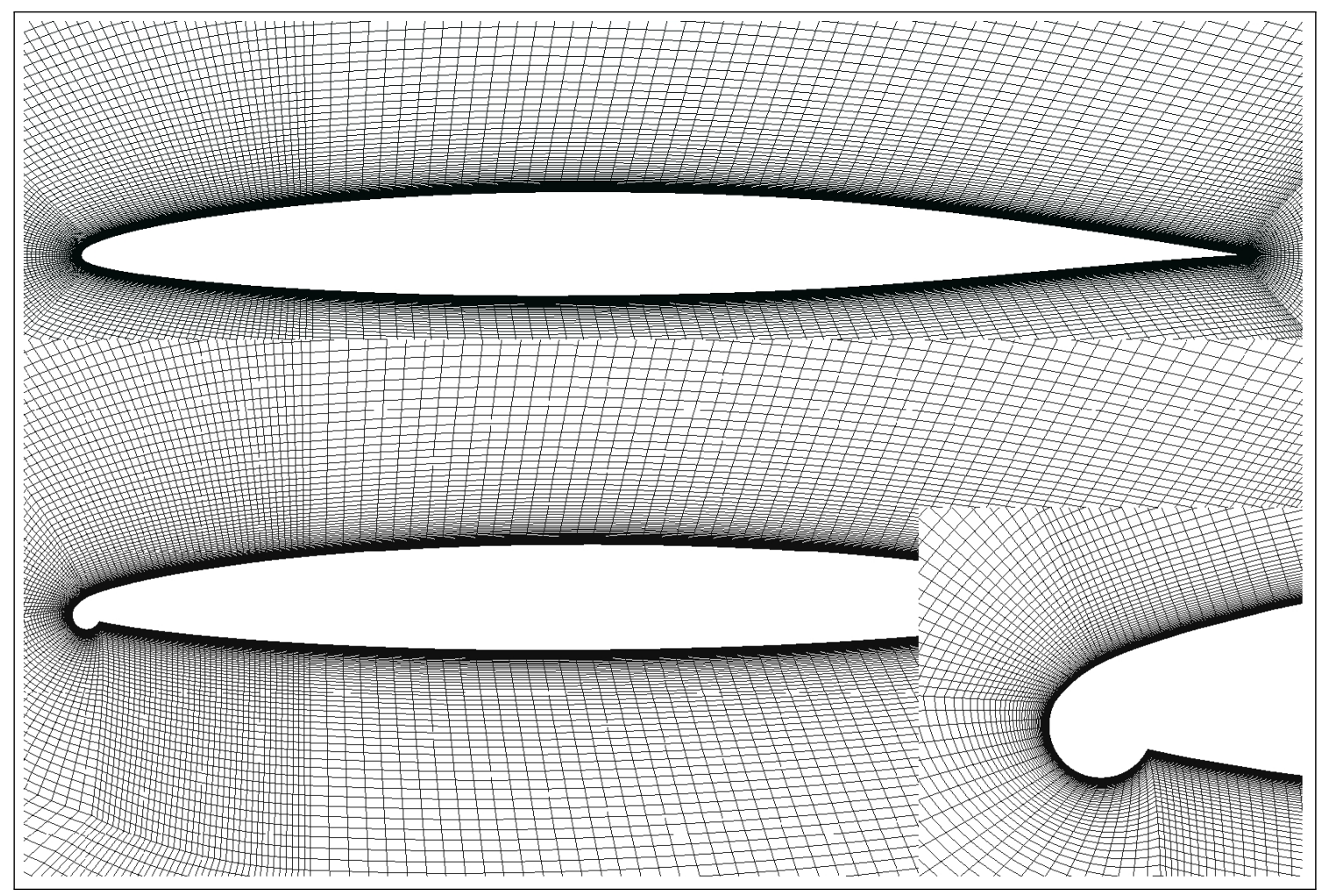

Fig. 5 Grids generated for the TsAGI-9140 airfoil and for its 1.5\% leading edge modification.

Boussinesq assumption as follows:

$$
\tau_{i j}=2 \mu_{t}\left(S_{i j}-\frac{1}{3} \frac{\partial u_{k}}{\partial x_{k}} \delta_{i j}\right)-\frac{2}{3} \rho k \delta_{i j}
$$

\section{Turbulence Model}

The SA turbulence model [15], which is commonly used in aeronautical applications and was specifically developed for external aerodynamics involving adverse pressure gradients, is employed in this study. The SA model solves for the modified turbulent viscosity $\hat{v}$ and is described by the following partial differential equation:

$$
\frac{\partial \hat{v}}{\partial t}+u_{j} \frac{\partial \hat{v}}{\partial x_{j}}=C_{b 1}\left(1-f_{t 2}\right) \hat{S} \hat{v}-\left(C_{w 1} f_{w}-\frac{C_{b 1} f_{t 2}}{\kappa^{2}}\right) \frac{\hat{v}}{d^{2}}+\frac{1}{\sigma_{t}}\left[\frac{\partial}{\partial x_{j}}(v+\hat{v}) \frac{\partial \hat{v}}{\partial x_{j}}+C_{b 2} \frac{\partial \hat{v}}{\partial x_{i}} \frac{\partial \hat{v}}{\partial x_{i}}\right]
$$

The turbulent viscosity is then obtained from

$$
v_{t}=\rho \hat{v} f_{v 1}
$$

where

$$
f_{v 1}=\frac{X^{3}}{X^{3}+C_{v 1}^{3}}
$$


and

$$
X=\frac{\hat{v}}{v}
$$

The model constants are given in Table 2. In Eq. (4), the production of turbulence is described by the term

$$
C_{b 1}\left(1-f_{t 2}\right) \hat{S} \hat{v}
$$

the diffusion of turbulence is described by the term

$$
\frac{1}{\sigma_{t}}\left[\frac{\partial}{\partial x_{j}}(v+\hat{v}) \frac{\partial \hat{v}}{\partial x_{j}}+C_{b 2} \frac{\partial \hat{v}}{\partial x_{i}} \frac{\partial \hat{v}}{\partial x_{i}}\right]
$$

and the destruction of turbulence is described by the term

$$
\left(C_{w 1} f_{w}-\frac{C_{b 1} f_{t 2}}{\kappa^{2}}\right) \frac{\hat{v}}{d^{2}}
$$

Table 2 SA turbulence model coefficients

\begin{tabular}{ccccccccc}
\hline \hline$\sigma_{t}$ & $C_{b 1}$ & $C_{b 2}$ & $\kappa$ & $C_{w 1}$ & $C_{w 2}$ & $C_{w 3}$ & $C_{v 1}$ & $C_{t 1}$ \\
\hline $2 / 3$ & 0.1355 & 0.622 & 0.41 & $C_{b 1} / \kappa^{2}+\left(1+C_{b 2}\right) / \sigma_{t}$ & 0.3 & 2 & 7.1 & 1 \\
\hline \hline
\end{tabular}

\section{Numerical Setup}

For the purpose of simulation in this paper, the transient semi-implicit pressure-linked equations (SIMPLE) algorithm was used, with the Courant-Friedrichs-Lewy (CFL) number being limited to the range 1-50. This solver is efficient, as a larger time step can be used while maintaining solution accuracy. It is a pseudo-solver with the use of inner loop iterations until convergence to the desired tolerance within each physical time step before proceeding to the next step. For low angles of attack, $\alpha<15 \mathrm{deg}$, steady-state simulations were conducted using the SimpleFOAM solver, which utilizes the SIMPLE algorithm. For large angles of attack, where flow separation was expected, unsteady simulations were performed with the pseudo-solver, i.e., transientSimpleFOAM. The unsteadiness in flow quantities at each time step was linearized by employing multiple correctors depending on the CFL number.

For time integration, a second-order-accurate Euler backward scheme was employed. The gradients of the flow parameters were estimated with the Gauss linear scheme for pressure and the least-squares method for other flow parameters. The Gauss linear upwind scheme, which is of second-order accuracy, was employed for the divergence of vectors and scalar flow field quantities.

The "linear upwind" scheme for divergence in OpenFOAM seems to produce more realistic results in $C_{L}$ coefficient 
convergence in comparison with the "limitedLinearV" scheme. The use of limited schemes for damping the divergence processes in flux quantities leads to an amplitude reduction in the transient convergence process for lift coefficient $C_{L}$, resulting in a lower level of the lift coefficient and earlier stall. Such effects of employing limiters in gradients and fluxes can be found in the NASA Technical Report [18]. Based on the above observations, all further simulations presented in this paper were made without the use of limited schemes.

\section{Results and Discussion}

Static aerodynamic hysteresis on a rectangular wing with an aspect ratio of $A R=5$ and TsAGI-9140 aerodynamic airfoil was detected in the variable-density wind tunnel T-106 in TsAGI at relatively high Reynolds numbers $R e=$ 4.85-5.65 $\times 10^{6}[9]$. The test section in this wind tunnel is bounded by a cylindrical perforated wall with a diameter of $D=2.4 \mathrm{~m}$, while the span of the wing was $b=1.5 \mathrm{~m}$. It can be expected that the developed separation flow on the wing was affected by the perforated wall of the test section and this interference should be taken into account when comparing the calculated and experimental results. To avoid this complication, the computational analysis in this paper was performed for a two-dimensional aerodynamic profile TsAGI-9140. To validate the selected OpenFOAM's numerical setup especially for stall prediction capability, the next sub-section examines a case study for two-dimensional flow around NACA 0012 airfoil, taken from [19, 20]. The Reynolds number for this case is $R e=6 \times 10^{6}$, which is close to the testing conditions at TsAGI.

\section{A. Validation of OpenFOAM for NACA 0012 airfoil at $R e=6 \times 10^{6}$}

The numerical setup presented in the previous section was validated against available experimental and computational results for the lift coefficient and pressure distribution from [19, 20] when NACA 0012 airfoil was tested at $R e=6 \times 10^{6}$. The major attention was given to OpenFOAM's prediction capabilities in the stall region. In this validation study, the SA turbulence model was considered along with the SST $k-\omega$ turbulence model.

Fig. 6 shows the computational prediction of the lift coefficient by the OpenFOAM along with with the prediction of the CFL3D code [19] and with the experimental results from [20]. The OpenFOAM simulations were using the URANS equations with the SA and SST $k$ - $\omega$ turbulence models at $R e=6 \times 10^{6}$ and $M=0.15$. The second-order accurate Euler backward scheme was used to compute derivative and advection terms. The first-order accurate Gauss scheme was used for turbulent terms in the start and after about 100-time steps for the rest of the simulation changed to the second-order accurate Gauss scheme to calculate the turbulence fields. The grid used has 80, 000 elements which fall in between grid level 2 and 3 for NACA 0012 airfoil in [19]. The experimental data, [19, 20], are given for test conditions with sandpaper strips on the leading edge of the airfoil with grain numbers of 80, 120 and 180, which indicate the number of grains per linear inch defining their size.

For angles of attack $\alpha<15^{\circ}$ predictions of the lift coefficient made by the OpenFOAM show good agreement with 


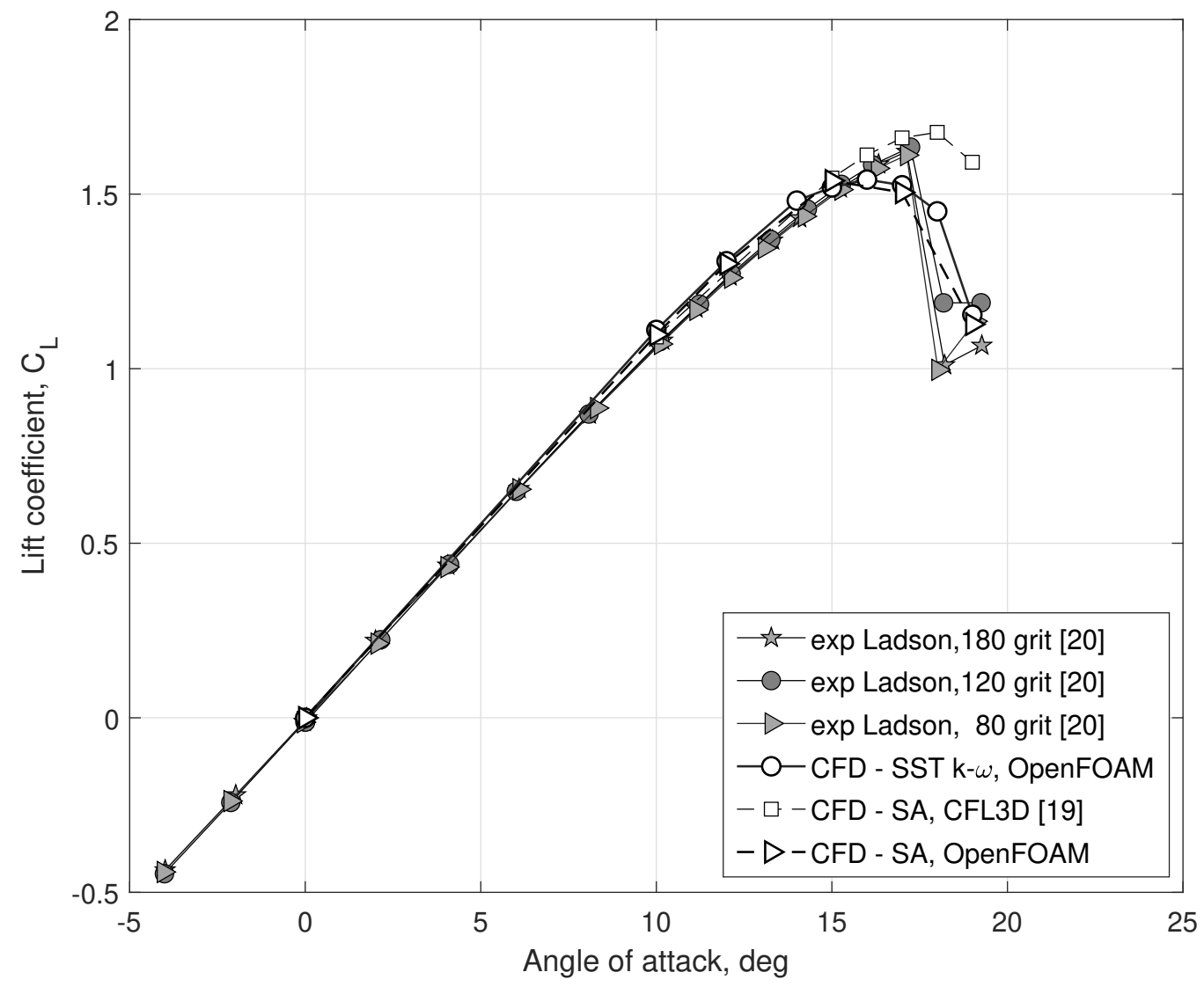

Fig. 6 Validation of the OpenFOAM: the lift coefficient $C_{L}$ of NACA 0012 at $R e=6 \times 10^{6}$ and $M=0.15$.

the NASA CFL3D code and experimental data [20]. At higher angles of attack $\alpha>15^{\circ}$ the OpenFOAM results for both SA and SST turbulence models go below the CFL3D data and follow more closely to the experimental data (see Fig. 6). This may be explained by the more early flow separation which starts from the trailing edge, both in the experiment and the OpenFOAM simulations when compared to the CFL3D simulation results. The OpenFOAM prediction for the maximum lift coefficient $C_{L_{\max }}$ is slightly lower of the level both in the experiment and the CFL3D simulation but at $\alpha=18^{\circ}$ and $\alpha=19^{\circ}$ the OpenFOAM results reasonably well follow the drop in the lift coefficient $C_{L}$ observed in the experiment [19, 20].

Computational predictions and experimentally measured values for pressure coefficient $C_{p}$ on suction and pressure sides of the airfoil are shown in Fig. 7 for conditions with $R e=6 \times 10^{6}$ and $\alpha=15^{\circ}$, which is approximately $2^{\circ}$ below the $C_{L_{\max }}$ point. A comparison of the distributions of $C_{p}$ shows that the OpenFOAM simulation dependencies are close to the results of CFL3D [19], and also closely match the experimental data from [20, 21].

For large Reynolds numbers, one can expect that the flow separation is more determined by the separation process, progressing forward from the trailing edge. This assumption is partly confirmed by the coefficient of skin friction, 


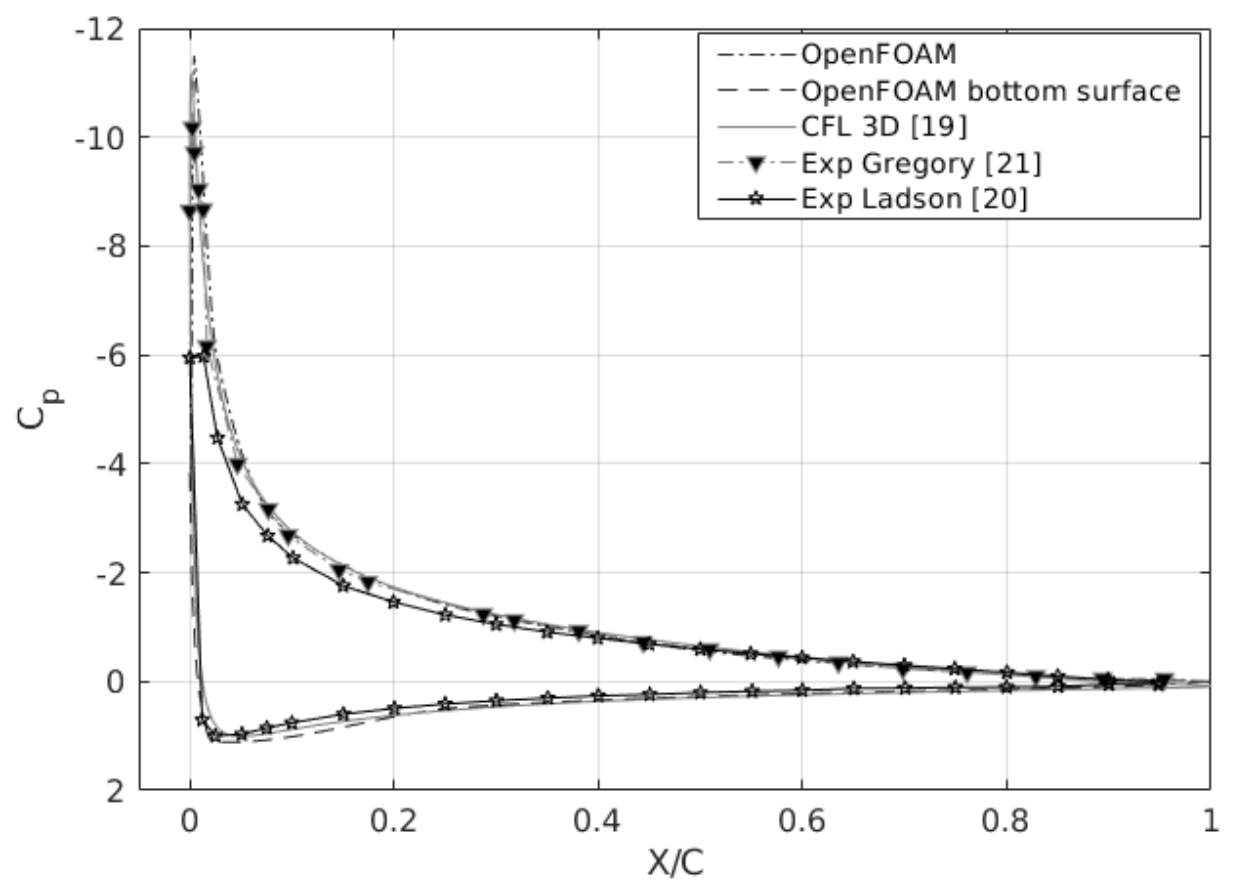

Fig. 7 Experimental and computational results for pressure coefficient $C_{p}$ on the NACA 0012 airfoil at $R e=$ $6 \times 10^{6}$ and $\alpha=15^{\circ}$.

$C_{f}$, on the suction side of the aerodynamic profile NACA 0012, predicted by the CFL3D code [19] and also by the OpenFOAM (see Fig 8). In a two-dimensional flow, separation from a smooth surface is characterized by a change in the sign of the skin friction coefficient from positive to negative $C_{f}<0$, since behind the separation point the flow velocity in the immediate vicinity of the surface changes to the opposite. A comparison of OpenFOAM and CFL3D predictions shows that the only separation point is very close to the trailing edge with a slight difference in its location, for example, $x_{s e p}^{O F}=0.903 c$ and $x_{s e p}^{C F L 3 D}=0.913 c$ (see Fig. 8). A small difference in the position of the flow separation points at $\alpha=15^{\circ}$ indicates that in the OpenFOAM simulation, the flow separates from the trailing edge earlier and with the increase of angle of attack continues to stay ahead of the separation point predicted in the CFL3D simulation. This assumption explains a significant difference in the lift coefficient, for example, at $\alpha=19^{\circ}$ the difference is $C_{L}^{C F L 3 D}-C_{L}^{O F}=0.45$. It can also be stated that there are no signs of existence of a laminar separation bubble on the suction side of the airfoil because there is no zone with the reverse flow defined by $C_{f}<0$ at the given angle of attack and Reynolds number.

Taking into account the above validation of the OpenFOAM computational setup, the same approach in the next subsection is applied to the aerodynamic profile TsAGI-9140. 


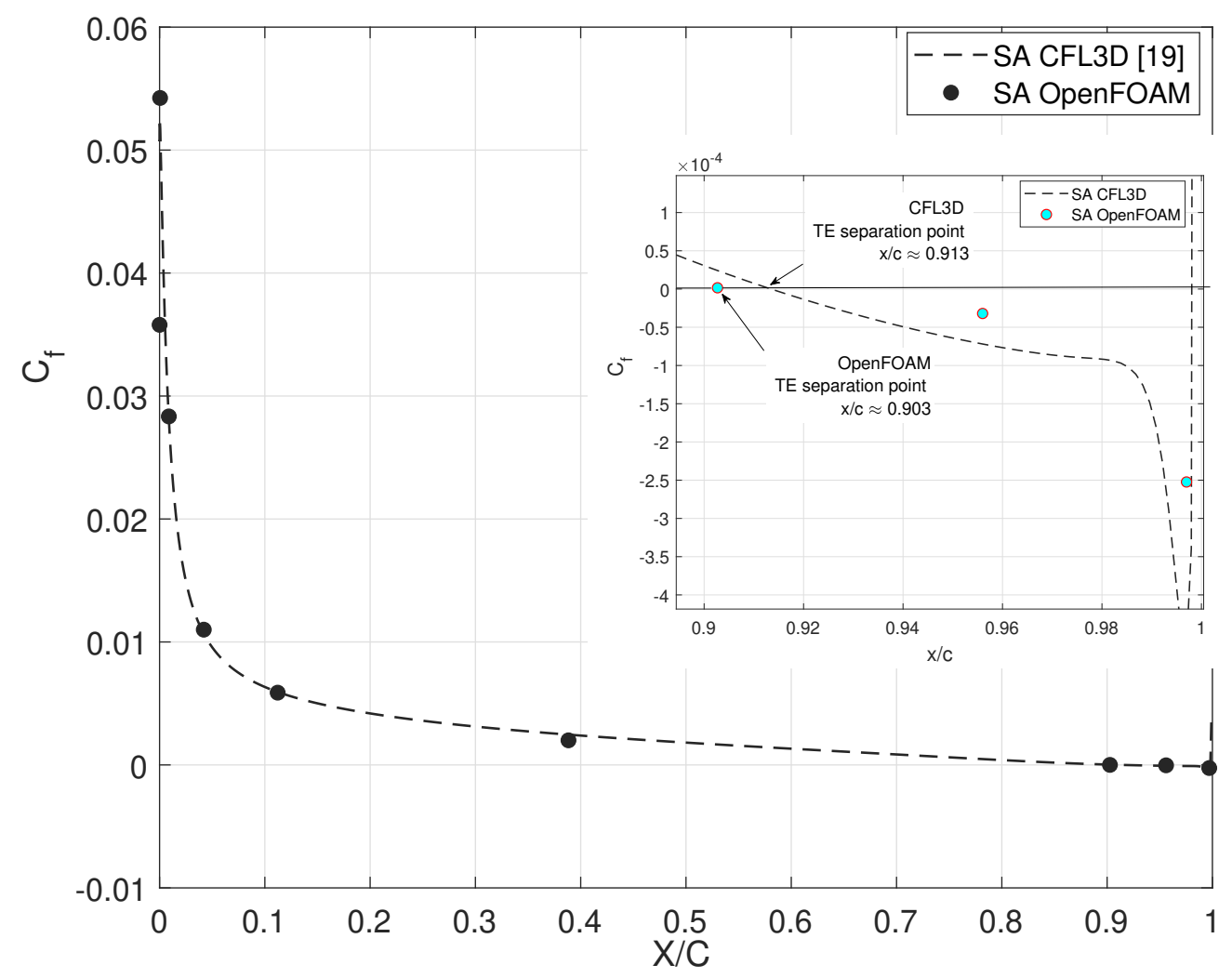

Fig. 8 Comparison of $C_{f}$ distribution for NACA0012, $R e=6 \times 10^{6}$ at $\alpha=15^{\circ}$

\section{B. OpenFOAM simulation results for TsAGI-9140 airfoil}

The computational structure described in section III was used to simulate the phenomenon of static hysteresis in the two-dimensional flow around the TsAGI-9140 aerodynamic profile. Before the final simulation, a preliminary assessment of grid convergence and force coefficients was carried out.

\section{Grid Convergence Study}

A grid convergence evaluation was performed to verify that the flow solutions are grid-independent. The number of elements in each grid and the converged lift coefficient are given in Table 3 , The data from the table are plotted in Fig. 9 As can be seen, there is a very minor change in the lift coefficient ( $<10$ counts) between the medium and fine grids for both $\alpha=10 \mathrm{deg}$ and $\alpha=13 \mathrm{deg}$. Therefore, to save computational resources, the medium grid with 45,000 elements was used for the remaining simulations.

\section{Time-Accurate Force Convergence Study}

The medium grid with 45, 000 elements was used to verify the convergence process in URANS simulations for two different angles of attack, namely, $\alpha=13 \mathrm{deg}$ and $17 \mathrm{deg}$ for the airfoil with $2 \%$ leading edge modification. Figure 10 
shows the convergence process for the lift force coefficient. It is evident that at a physical time of $0.15 \mathrm{~s}$, the lift coefficient has practically converged. Therefore, the above setup was used to initiate and carry out the remaining CFD simulations.

Table 3 Lift coefficient grid convergence study

\begin{tabular}{llll}
\hline \hline Number of elements & 30,000 & 45,000 & 60,000 \\
$C_{L}$ at $\alpha=10 \mathrm{deg}$ & 1.1028 & 1.1132 & 1.1090 \\
$C_{L}$ at $\alpha=13 \mathrm{deg}$ & 1.1921 & 1.2050 & 1.2028 \\
\hline \hline
\end{tabular}

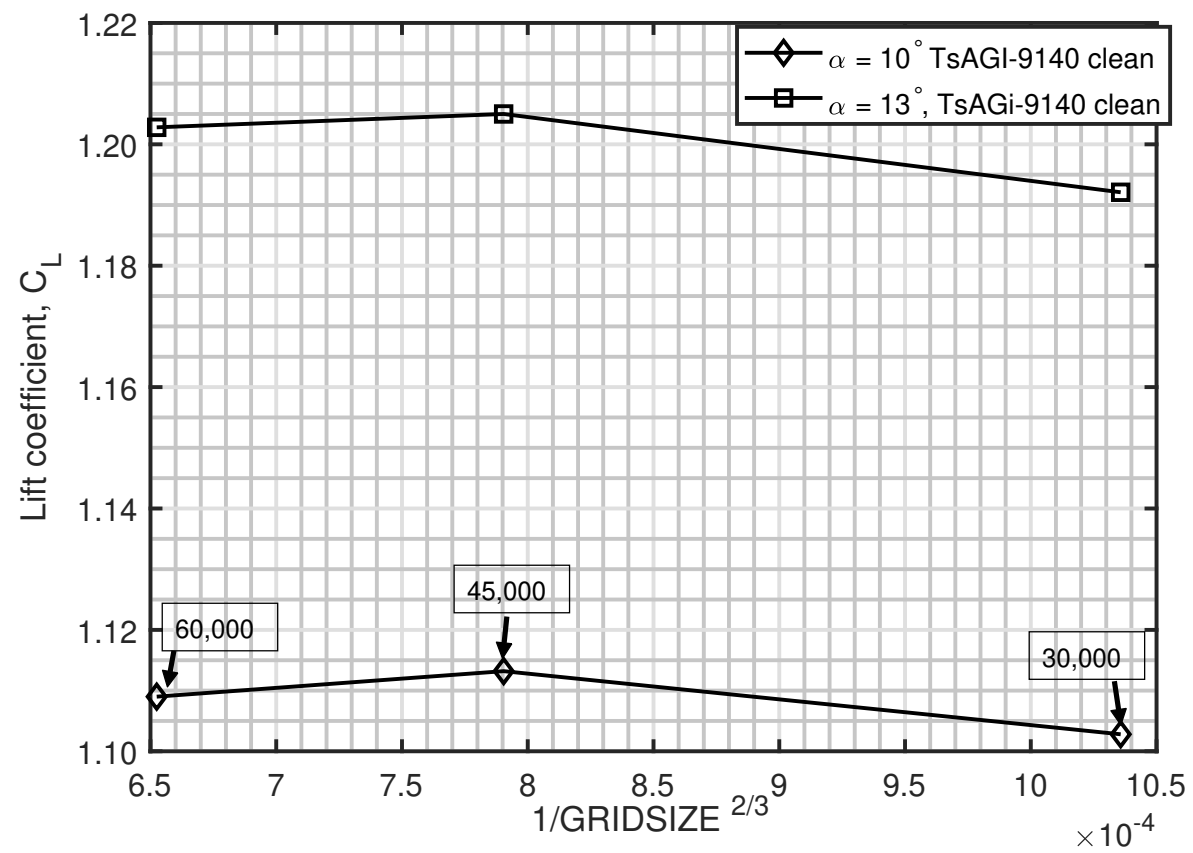

Fig. 9 Grid convergence study for the lift coefficient $C_{L}$.

\section{Computational Results for Static Hysteresis}

Figure 11 shows the computational prediction of the dependence of the lift coefficient on the angle of attack. The $C_{L}(\alpha)$ dependence includes noticeable static hysteresis loops. These results correspond to the airfoil in its clean configuration and also with $1.5 \%$ and $2 \%$ modifications of the leading edge. The clean airfoil has a maximum lift coefficient $C_{L \max } \approx 1.2$ and stalls at $\alpha=13.5 \mathrm{deg}$. The sharp drop in the lift coefficient on the top branch from $C_{L \max } \approx 1.2$ to $C_{L} \approx 0.8669$ on the bottom branch indicates the possible existence of a static hysteresis loop. When tested with a reduction of angle of attack in steps of $1 \mathrm{deg}$, a 2-deg-wide hysteresis loop was identified. It should be noted that for the clean airfoil, no static hysteresis loop was identified in the experiment (see Fig. 47). It is not clear that the experimental study actually involved the reverse change of angle of attack for the clean airfoil, as the main objective 


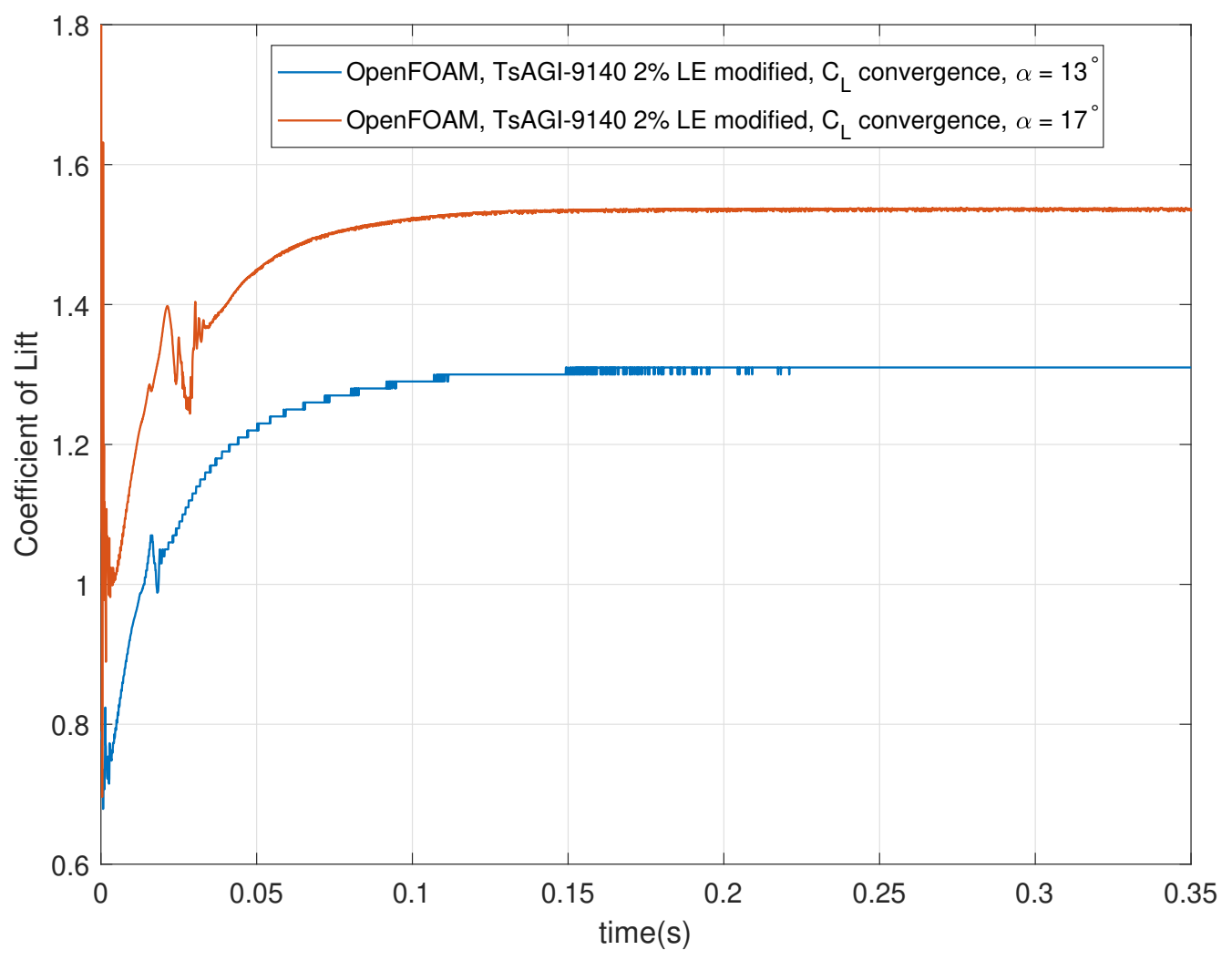

Fig. 10 Convergence process of the lift coefficient $C_{L}$.

was to modify the wing leading edge and increase the maximum lift coefficient.

The results for the modified airfoils demonstrate a significant increase in the maximum value of the lift coefficient $C_{L \max }$ along with a delay in the angle of attack, corresponding to the maximum lift point $\alpha_{C_{L \max }}$, similar to the wind tunnel test results for the finite-aspect-ratio wing $(A R=5)$ shown in Fig. 4.

For the $1.5 \%$ leading edge modification, the maximum lift coefficient is $C_{L \max } \approx 1.68$, and flow stalls from the top branch at $\alpha=22 \mathrm{deg}$. The $2 \%$ modification increases the maximum lift coefficient slightly to $C_{\text {Lmax }} \approx 1.733$, and the delay in stall angle of attack is also increased slightly to $\alpha_{C_{L \max }}=22.5 \mathrm{deg}$. In comparison with the aerodynamic performance of the clean airfoil, the 2\% modification brings about a 9-deg delay in stall angle $\alpha_{s}$. For the $1.5 \%$ and $2 \%$ leading edge modified airfoils, the very abrupt and sharp jump in the lift coefficient from $C_{L \max } \approx 1.7$ to $C_{L} \approx 0.9$ indicates a high possibility of static hysteresis. On testing to capture static hysteresis, significant loops were obtained in the computational data. The static hysteresis loops were approximately $5.5 \mathrm{deg}$ wide, and the return to the top branch occurred at $\alpha \approx 17 \mathrm{deg}$. 


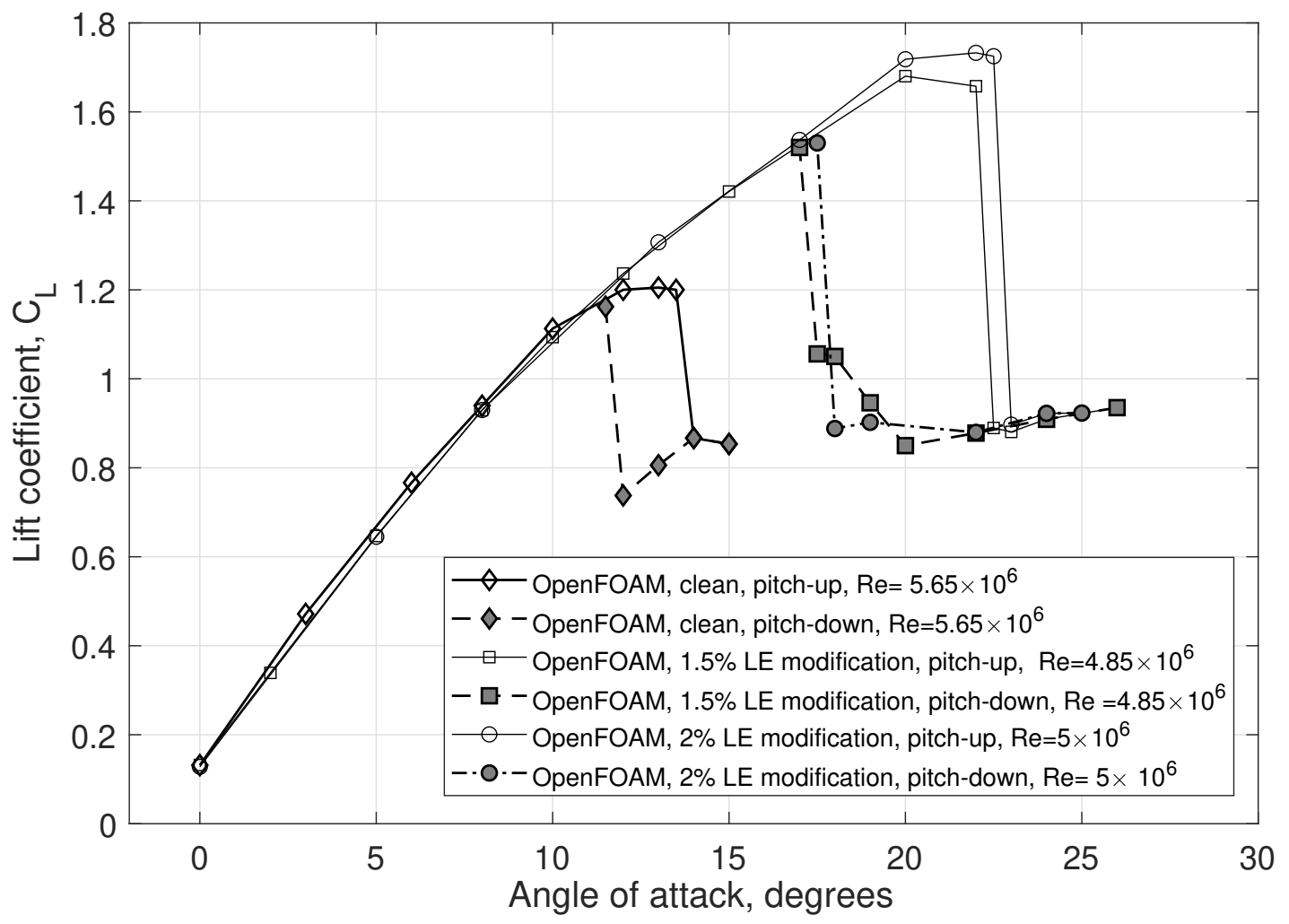

Fig. 11 Lift coefficient hysteresis dependences $C_{L}(\alpha)$ at $R e \approx 5 / 5.65 \times 10^{6}$.

\section{Flowfield Visualization}

Visualization of the flow field for the velocity distribution with superimposed streamlines on the top and bottom branches of static stall hysteresis helps to understand the physical nature of this phenomenon. Figure 12 shows the flow field around the clean airfoil for three angles of attack $\alpha=8 \mathrm{deg}, 12 \mathrm{deg}$, and $14 \mathrm{deg}$. At $\alpha=8 \mathrm{deg}$, the flow is separated in the middle of the upper surface. The development of such separated circulatory zone starts from the trailing edge. At $\alpha=14 \mathrm{deg}$, the flow is fully separated from the leading edge with periodically shedding downstream vortices inducing buffet in the form of small-amplitude oscillations in the lift coefficient.

When $\alpha=12 \mathrm{deg}$, there are two bistable flow structures (Figs. $12 \mathrm{~b}$ and $12 \mathrm{~d}$ ). The flow pattern on the top branch of static hysteresis (Fig. 12p) shows the buffet process with shedding downstream large-scale vortices generated on the leading edge of the airfoil. Figure $12 \mathrm{p}$ shows the flow pattern at the moment when the large vortex is passing just above the airfoil surface and generating the maximum value of the lift coefficient $C_{L}$. This kind of flow is often observed for thin airfoils under stall conditions [22-24]. The flow pattern on the bottom branch of static hysteresis (Fig. [12 d) shows an open circulatory zone with a lower level of unsteadiness in the flow.

Figures 13 and 14 show the flow patterns for the $1.5 \%$ leading edge modification for the top and bottom branches of the static hysteresis, respectively. The trailing edge separation starts at approximately $\alpha=8 \mathrm{deg}$, and the separation 


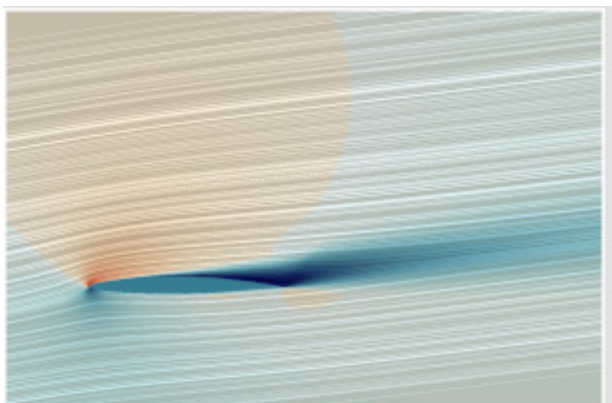

a) $\alpha=8 \mathrm{deg}$ (top branch)

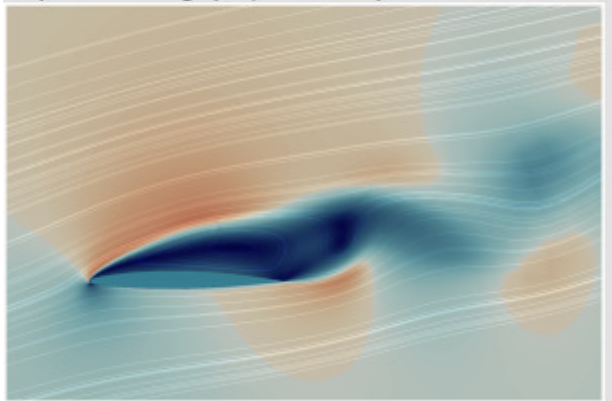

c) $\alpha=14 \mathrm{deg}$ (bottom branch)

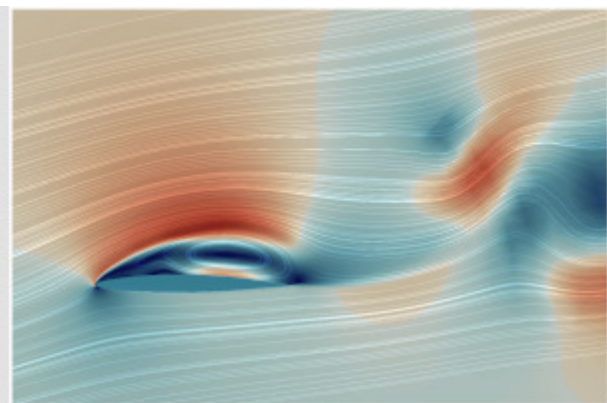

b) $\alpha=12 \operatorname{deg}$ (top branch)

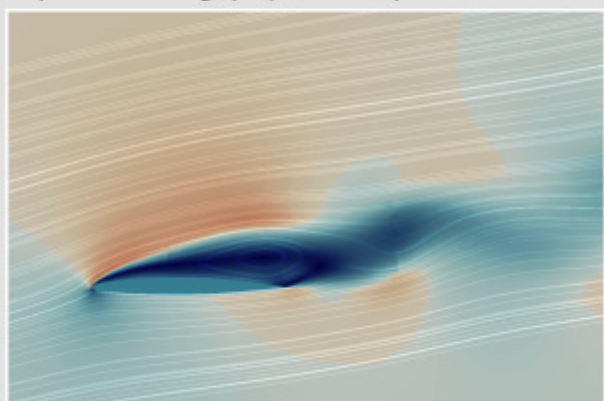

d) $\alpha=12 \mathrm{deg}$ (bottom branch)

\begin{tabular}{llllll}
\hline 0 & 20 & $40 \quad 60$ & 80 & 100 & 120
\end{tabular}

Fig. 12 Flow field patterns around the clean TsAGI-9140 airfoil configuration.

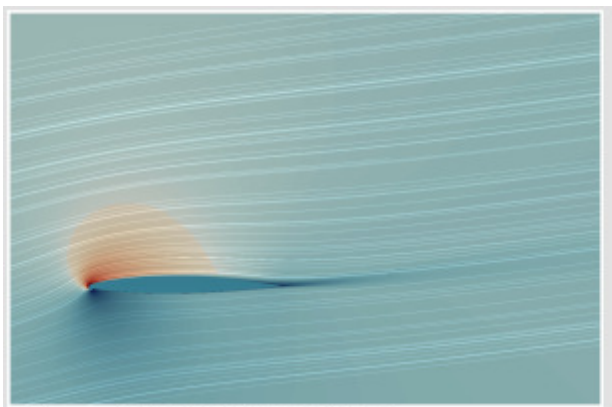

a) $\alpha=10 \mathrm{deg}$ (top branch)

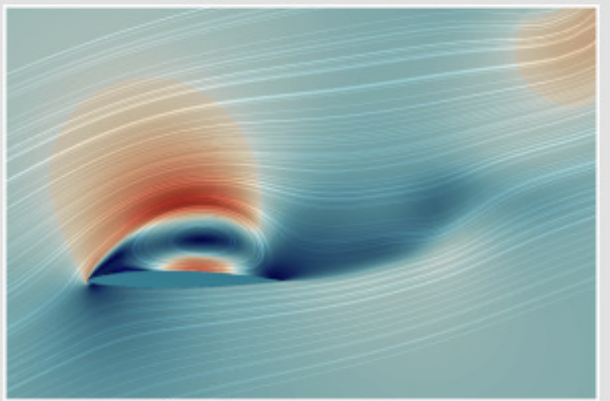

c) $\alpha=20 \mathrm{deg}$ (top branch)

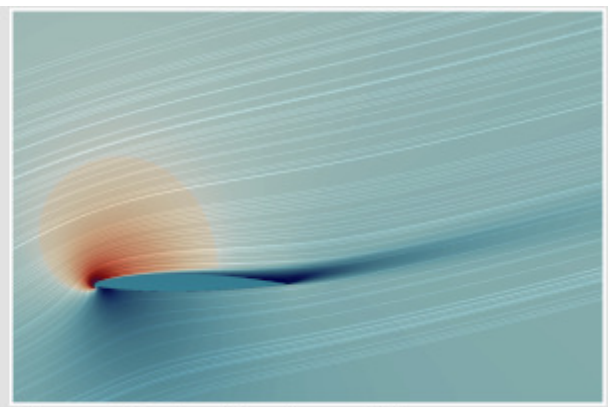

b) $\alpha=18 \mathrm{deg}$ (top branch)

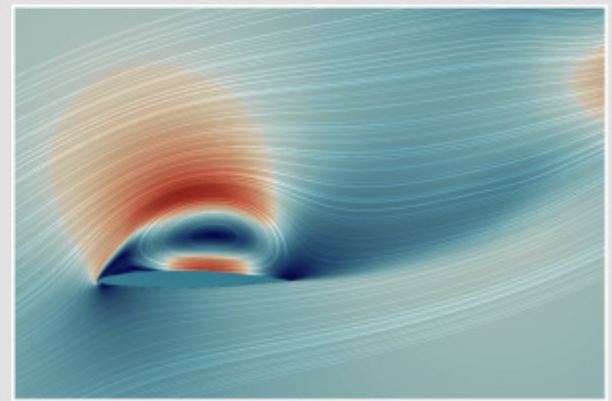

d) $\alpha=22 \mathrm{deg}$ (top branch)

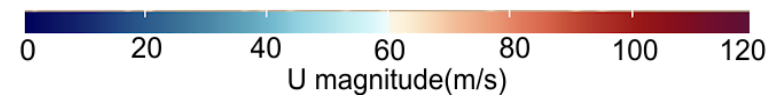

Fig. 13 Flow field patterns for the $1.5 \%$ modification on the top branch of static hysteresis. 


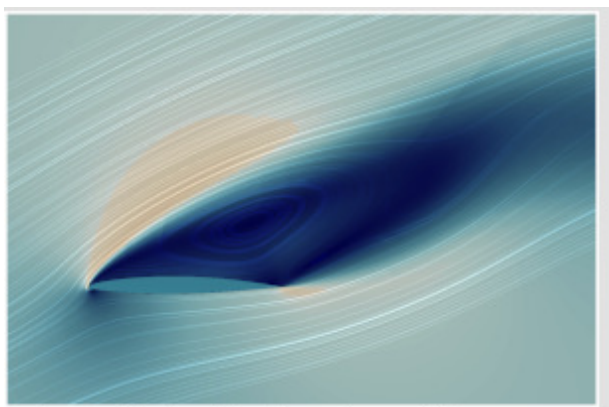

a) $\alpha=24 \mathrm{deg}$ (bottom branch)

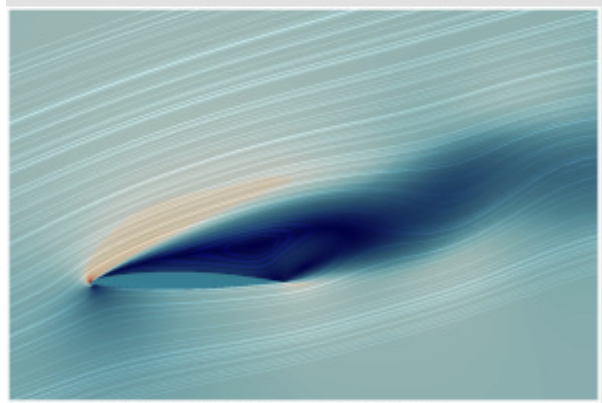

c) $\alpha=18 \mathrm{deg}$ (bottom branch)

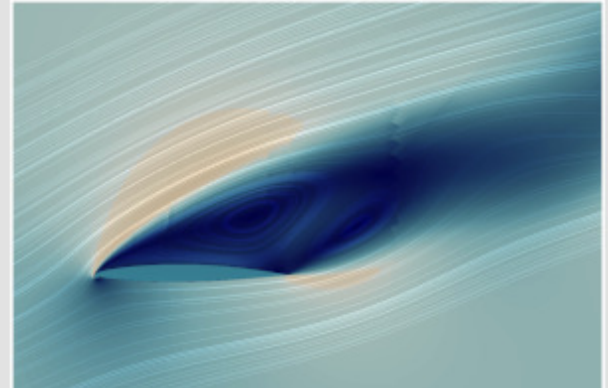

b) $\alpha=22 \operatorname{deg}$ (bottom branch)

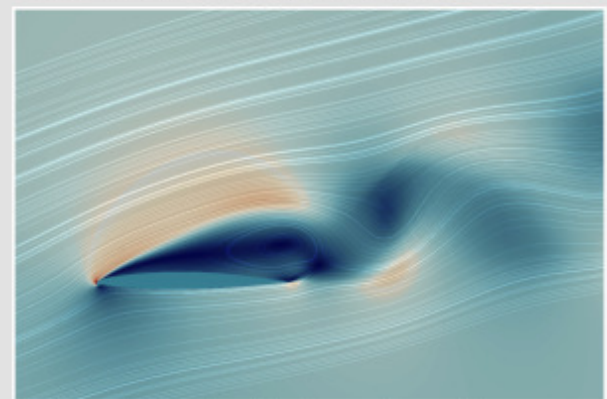

d) $\alpha=17.5 \mathrm{deg}$ (bottom branch)

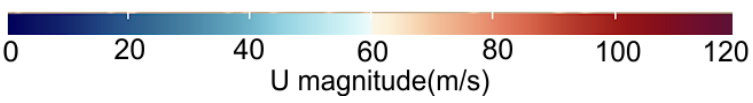

Fig. 14 Flow field patterns for the $1.5 \%$ modification on the bottom branch of static hysteresis.

point gradually propagates toward the leading edge (Figs. 11, 13, and 13 ). At $\alpha=18 \mathrm{deg}$, the separation point is located roughly in the middle of the upper surface, leaving behind a small steady circulatory zone. With increasing angle of attack, when $\alpha \geq 20 \mathrm{deg}$, the separated flow on the top branch of static hysteresis becomes highly agitated. When the angle of attack exceeds $\alpha=22 \mathrm{deg}$, the flow transits to a fully separated circulatory zone connected to the leading and trailing edges (Fig. [14). These flow conditions correspond to the bottom branch of the static hysteresis shown in Fig. 11. The buffet intensity on the bottom branch has a much lower level. Figures 14 a $14 \mathrm{~d}$ show the gradual transformation of the separated flow pattern as the angle of attack decreases from $\alpha=24 \mathrm{deg}$ to $17.5 \mathrm{deg}$.

Comparison of the velocity distributions on the upper and lower branches of the static hysteresis, shown in Fig. 13 and 14. clearly demonstrates higher velocities above the suction side of the airfoil for regimes on the upper branch of the static hysteresis (almost double the upward flow velocity). This leads to larger pressure differences across the airfoil for flow structures on the upper branch of the static hysteresis and so indicates the origin of the static hysteresis loop.

Periodic variations of the lift coefficient on the top and bottom branches of the static hysteresis loop at $\alpha=22$ deg are shown in Fig. 15. The black curve represents high-amplitude buffet with average value $C_{L \text { av }} \approx 1.65$ (see the left-hand axis), and the red line represents small-amplitude buffet on the bottom branch of the static hysteresis with average value $C_{L \mathrm{av}} \approx 0.88$ (see the right-hand axis). Note that the aerodynamic buffet has a higher frequency and a lower amplitude on the bottom branch of static hysteresis. The presented flow patterns of the vorticity distribution highlight how the flow 


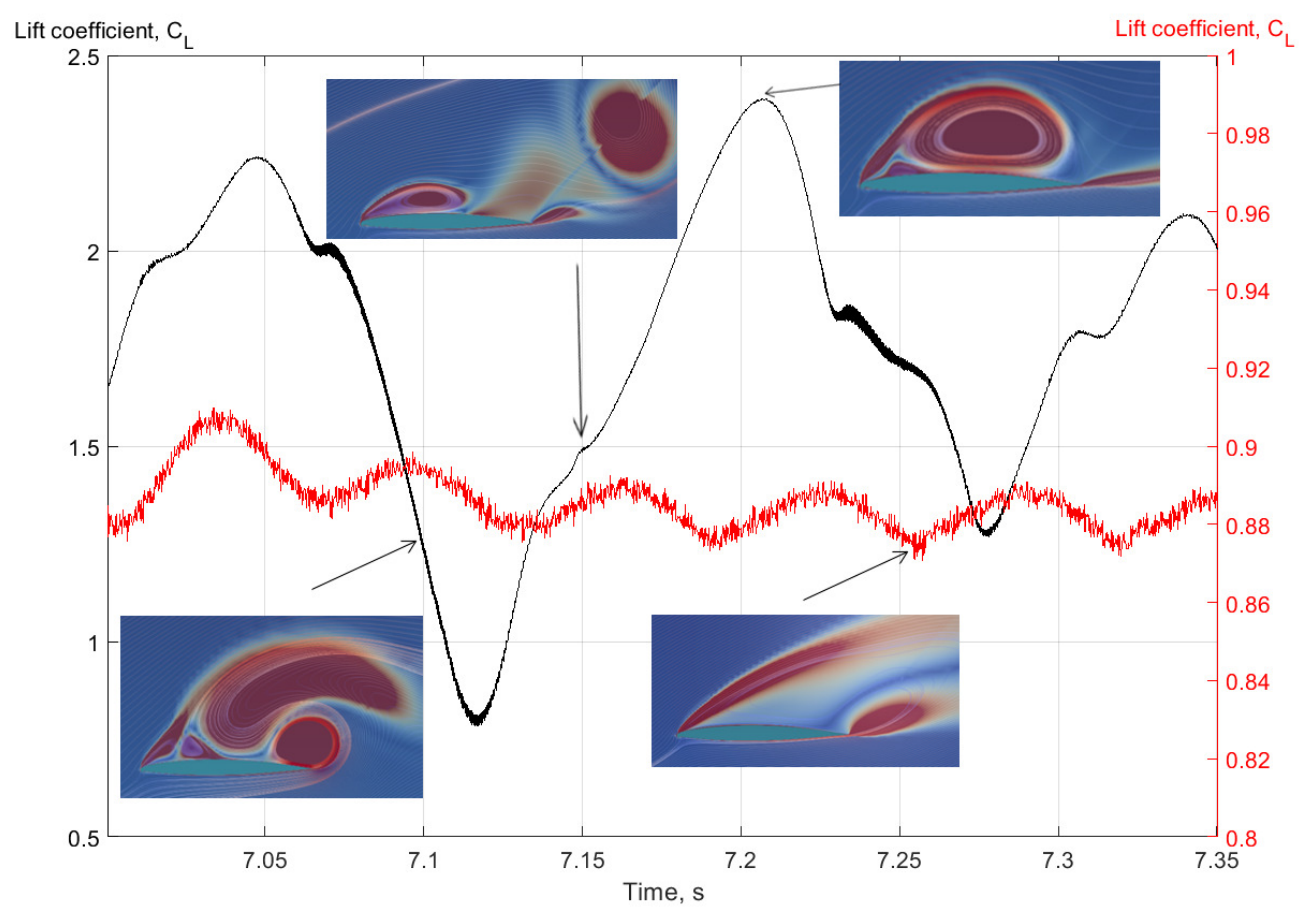

Fig. 15 Vorticity distributions and variations of the lift coefficient $C_{L}(t)$ for the top and bottom branches of static hysteresis at $\alpha=22 \mathrm{deg}$.

pattern changes at different phases of the oscillatory buffet.

\section{Conclusions}

Computational simulations of stall aerodynamics for the thin asymmetric TsAGI-9140 airfoil and its modifications that increase the curvature of the leading edge have been performed for relatively high Reynolds number $\operatorname{Re} \approx 6 \times 10^{6}$ using the OpenFOAM. The results of these simulations show a considerable increase in the maximum lift coefficient, $C_{L \max }$, roughly on $40 \%$ with the increase of the leading edge curvature. This increase is accompanied by enlargement of the static aerodynamic hysteresis loops. The two-dimensional simulation results are found in good qualitative agreement with the TsAGI wind tunnel test data for a finite-aspect-ratio wing with $A R=5$ in terms of the lift coefficient dependence on the angle of attack. The obtained simulation results and flow field visualizations help to better understand the source mechanisms of the static aerodynamic hysteresis phenomenon.

\section{References}

[1] Ericsson, L. E., and Reding, J. P., "Unsteady Airfoil Stall,” Tech. Rep. CR-66787, NASA, 1969.

[2] Ekaterinaris, J. A., and Platzer, M. F., "Computational Prediction of Airfoil Dynamic Stall,” Progress in Aerospace Sciences, Vol. 33, No. 11-12, 1997, pp. 759-846. https://doi.org/https://doi.org/10.1016/S0376-0421(97)00012-2 
[3] Cummings, R. M., and Goreyshir, M., "Challenges in the Aerodynamic Modelling of an Oscillating and Translating Airfoil at Large Incidence Angles," Aerospace Science and Technology, Vol. 28, No. 1, 2013, pp. 176-190. https://doi.org/https: //doi.org/10.1016/j.ast.2012.10.013

[4] Abramov, N. B., Goman, M. G., Khrabrov, A. N., and Soemarwoto, B. I., "Aerodynamic Modeling for Poststall Flight Simulation of a Transport Airplane," Journal of Aircraft, Vol. 56, No. 4, 2019, pp. 1427-1440. https://doi.org/https: //doi.org/10.2514/1.C034790

[5] Timmer, W. A., “Two-Dimensional Low-Reynolds Number Wind Tunnel Results for Airfoil NACA 0018," Wind Engineering, Vol. 32, No. 6, 2008, pp. 525-537. https://doi.org/https://doi.org/10.1260/030952408787548848

[6] Williams, D. R., Reisner, G., F., D., H., Vahl, and Strangfeld, C., "Modeling Lift Hysteresis with a Modified Goman-Khrabrov Model on Pitching Airfoils,” AIAA, Vol. 55, No. 2, 2017, pp. 403-410. https://doi.org/https://doi.org/10.2514/1.J054937.

[7] Mizoguchi, M., Kajikawa, Y., and Itoh, H., "Static Stall Hysteresis of Low-Aspect-Ratio Wings,” Tech. Rep. 32, AIAA Applied Aerodynamics conference, 2014.

[8] Hoffmann, J. A., "Effects of Freestream Turbulence on the Performance Characteristics of an Airfoil," AIAA, Vol. 29, No. 9, 1991, pp. 1353-1354. https://doi.org/https://doi.org/10.2514/3.10745

[9] Svischev, G. P., "Investigation of a Low Drag Airfoil with Different Leading Edge Modifications for Increase of Maximum Lift Force,” Tech. Rep. TsAGi’s proceedings, TsAGi, 1946.

[10] Mittal, S., and Saxena, P., "Prediction of Hysteresis Associated with Static Stall of an Airfoil," AIAA, Vol. 38, No. 5, 2000, pp. 2179-2189. https://doi.org/https://doi.org/10.2514/2.1051

[11] Mittal, S., and Saxena, P., "Prediction of Hysteresis Associated with Static Stall of an Airfoil," Comp. Methods in App. Mech Engineering, Vol. 191, No. 19-20, 2002, pp. 2207-2217. https://doi.org/https://doi.org/10.1016/S0045-7825(01)00382-6.

[12] Sereez, M., Abramov, N. B., and Goman, M. G., "Computational Simulation of Airfoils Stall Aerodynamics at Low Reynolds Numbers,” Tech. Rep. Applied Aerodynamics Conference, RaES, 2016.

[13] Wauters, J., Degroote, J., and Vierendeels, J., "Comparative Study of Transition Models for High-Angle-of-Attack Behavior," AIAA, Vol. 57, No. 6, 2019, pp. 2356-2371. https://doi.org/https://doi.org/10.2514/1.J057249

[14] Ashton, N., and Skaperdas, V., "Verification and Validation of OpenFOAM for High-Lift Aircraft Flows," Journal of Aircraft, Vol. 56, No. 4, 2019, pp. 1641-1657. https://doi.org/https://doi.org/10.2514/1.C034918

[15] Spalart, P. R., and Allmaras, S. R., “A One-Equation Turbulence Model for Aerodynamic Flows,” Tech. Rep. 30th Aerospace Sciences Meeting and Exhibit, AIAA, 1992.

[16] OpenFOAM, “OpenFOAM: The Open Source Computational Fluid Dynamics Toolbox,” , . http://www.openfoam.com last accessed 8 June 2019. 
[17] Chen, G., Xiong, Q., Morris, J., P., Paterson, E., Sergeev, A., and Wang, Y. C., "A One-Equation Turbulence Model for Aerodynamic Flows," Notices of the AMS, Vol. 61, No. 4, 2014, pp. 354-363.

[18] Berger, M., Aftosmis, M. J., and Murman, “Analysis of Slope Limiters on Irregular Grids,” Tech. Rep. NAS-05-007, NASA, 2005.

[19] NASA, “2D NACA 0012 Airfoil Validation Case,”, , URL https://turbmodels.larc.nasa.gov/naca0012_val.html last accessed 25 June 2020.

[20] Ladson, C. L., "Effect of Independent Variation of Mach and Reynolds Numbers on the Low-Speed Aerodynamics Characteristics of the NACA 0012 Airfoil Section,” Tech. Rep. Technical Memorandum 4074, NASA, 1988. URLhttps://ntrs.nasa.gov/archive/ nasa/casi.ntrs.nasa.gov/19880019495.pdf

[21] Gregory, N., and O'Reilly, C. L., "Low Speed Aerodynamic Characteristics of NACA 0012 Aerofoil Section, Including the Effects of Upper-Surface Roughness Simulating Hoar Frost,” Tech. Rep. Research and Memorandum 3726, Areronautical Research Council, 1970. URL http://citeseerx.ist.psu.edu/viewdoc/download?doi=10.1.1.227.696\&rep=rep1\&type=pdf

[22] Berg, B. V. D., "Role of Laminar Bubbles in Airfoil Leading-Edge Stalls," AIAA, Vol. 19, No. 5, 1981 , pp. 553-570. https://doi.org/https://doi.org/10.2514/3.7798

[23] Visbal, M. R., and Garmann, D. J., "Analysis of Dynamic Stall on Pitching Airfoil Using High-Fidelity Large-Eddy Simulations," AIAA, Vol. 56, No. 1, 2018, pp. 46-63. https://doi.org/https://doi.org/10.2514/1.J056108

[24] Esfahani, A., Webb, N., , and Samimy, M., "Flow Separation Control over a Thin Post-Stall Airfoil: Effects of Excitation Frequency,” AIAA, Vol. 57, No. 5, 2019, pp. 1826-1837. https://doi.org/https://doi.org/10.2514/1.J057796 